\title{
Cerebral fat embolism syndrome after long bone fracture due to traffic accident: a case report
}

\author{
Xing-Yong Chen ${ }^{1, \#}$, Jian-Ming Fan ${ }^{2, \#}$, Ming-Feng Deng ${ }^{2}$, Ting Jiang ${ }^{3}$, Feng Luo ${ }^{3}$ \\ 'Department of Neurology, Fujian Provincial Hospital, Fujian Medical University Provincial Clinical College, Fuzhou 350001, China. \\ ${ }^{2}$ Intensive Care Unit, Fujian Provincial Hospital Wuyi Branch Hospital, Wuyishan City Hospital, Wuyishan 354300, China. \\ ${ }^{3}$ Department of Image Diagnoses, Fujian Provincial Hospital Wuyi Branch Hospital, Wuyishan City Hospital, Wuyishan 354300, China. \\ \#Authors contributed equally.
}

Correspondence to: Dr. Xing-Yong Chen, Department of Neurology, Fujian Provincial Hospital, Fujian Medical University Provincial Clinical College, Fuzhou 350001, China. E-mail: cxyong77@163.com; Dr. Jian-Ming Fan, Intensive Care Unit, Fujian Provincial Hospital Wuyi Branch Hospital, Wuyishan City Hospital, Wuyishan 354300, China. E-mail: 13706910979@139.com

How to cite this article: Chen XY, Fang JM, Deng MF, Jiang T, Luo F. Cerebral fat embolism syndrome after long bone fracture due to traffic accident: a case report. Neuroimmuno/Neuroinflammation2018;5:31. http://dx.doi.org/10.20517/2347-8659.2018.23

Received: 26 Apr 2018 First Decision: 11 Jun 2018 Revised: 20 Jun 2018 Accepted: 20 Jun 2018 Published: 1 Aug 2018

Science Editor: Athanassios P. Kyritsis Copy Editor: Jun-Yao Li Production Editor: Cai-Hong Wang

\begin{abstract}
Cerebral fat embolism syndrome (CFES) is an uncommon but serious complication of long bone fracture. We reported a 19-year-old male patient who sustained CFES due to multiple limbs long bone fractures after a traffic accident injury. He gradually developed into coma within $24 \mathrm{~h}$ after his injury. The arterial blood gas analyses were normal. There was a small amount of gas in the right pleural cavity on the thoracic computed tomography (CT). Although there were no remarkable intracranial abnormalities on the initial brain CT findings, the typical brain magnetic resonance imaging (MRI) findings of the starfield pattern and scattered foci were observed. Both T2-weighted imaging and diffusion weighted imaging of MRI indicated multiple scattered lesions in the bilateral cerebrum hemisphere white matter, grey matter, basal ganglia, corpus callosum and thalamus indicative of acute infarcts without microbleeding on the susceptibilityweighted imaging sequences. With the above findings, the diagnosis of the case was cerebral fat embolism syndrome. Although the patient was treated with comprehensive support in the intensive care unit, he remained unconscious and was discharged after 7 days of hospitalization.
\end{abstract}

Keywords: Cerebral fat embolism, infarction, long bone fracture

\section{INTRODUCTION}

Fat embolism syndrome (FES) is a potentially fatal complication and occurs most commonly after long bone fracture. Many cases occur as subclinical events and remain undiagnosed. The incidence of clinically

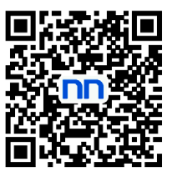




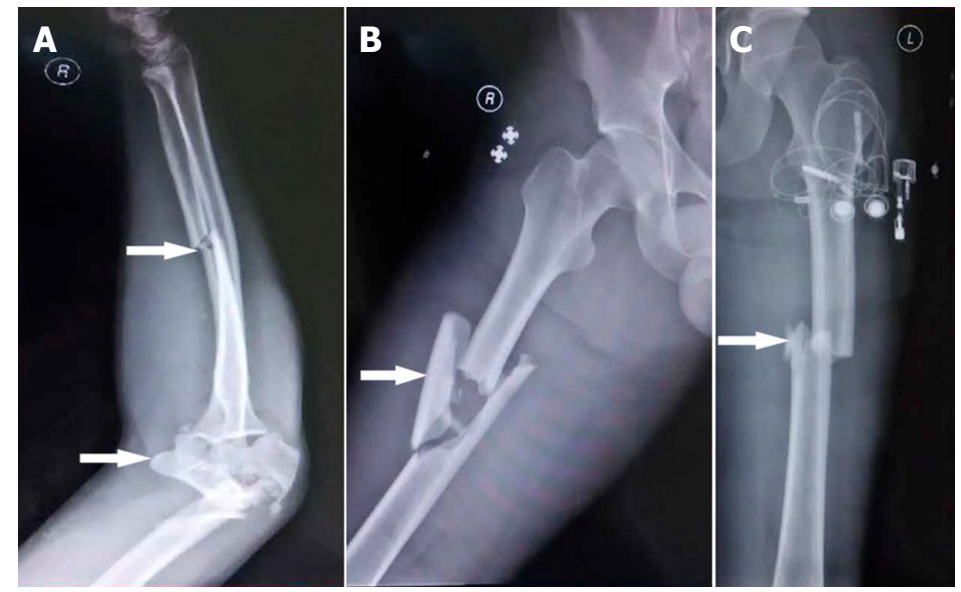

Figure 1. X-ray showed multiple long bone fractures, including right distal humerus distal, right ulna and its olecranon (A), bilateral femur shafts $(B, C)$ (arrow)

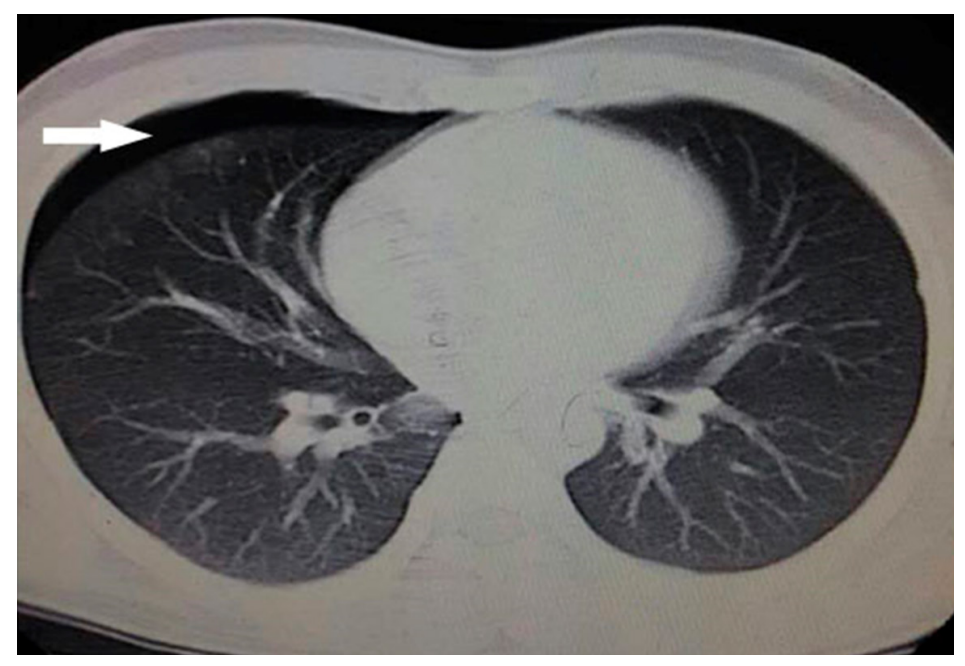

Figure 2. Thoracic computed tomography indicated a small amount of gas in the right pleural cavity (arrow)

significant FES occurs only in $0.9 \%-2.2 \%$ of long-bone fractures ${ }^{[1]}$. FES is characterized by various central nervous system, respiratory, cutaneous, and hematological manifestations ${ }^{[2]}$. Cerebral fat embolism syndrome (CFES) is a variant of FES. The neurological symptoms can be focal or diffuse, and most of the times exist with respiratory symptoms. We here reported a case of cerebral fat embolism (CFE) due to multiple long bone fractures of limbs after a traffic accident injury.

\section{CASE REPORT}

A 19-year-old male patient was admitted to the Emergency Department after a traffic accident injury on May 19, 2016. He could not move his limbs except for the left upper limb due to bone fractures. He had no history of loss of consciousness, ear, nose, and throat bleed, vomiting, or convulsion. He was conscious, oriented with normal breathing and was hemodynamically stable. In the physical examination, body temperature was $36.8^{\circ} \mathrm{C}$, pulse was $78 / \mathrm{min}$, breath rate was $22 / \mathrm{min}$, and blood pressure was $130 / 65 \mathrm{mmHg}$. There were not positive neurologic dysfunctions. Electrocardiogram was normal. X-ray showed multiple long bone fractures, including bilateral femurs, right distal humerus, right ulna and its olecranon [Figure 1]. There was a small amount of gas in the right pleural cavity on the thoracic computed tomography (CT) [Figure 2]. 


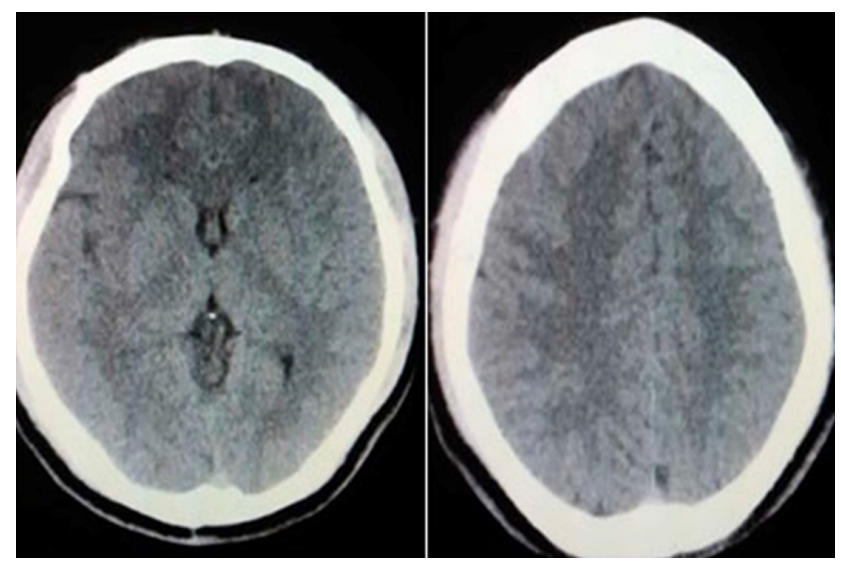

Figure 3. There was no remarkable abnormality on the brain computed tomography on admission

However, there were no remarkable abnormalities on abdominal CT and color Doppler ultrasound. The results of hematologic and biochemical parameters of the patient were as follows at first admission: hemoglobine $149 \mathrm{~g} / \mathrm{L}$, platelet count $187 \times 10^{9} / \mathrm{L}$, procalcitonin $0.10 \mathrm{ng} / \mathrm{mL}$, prothrombin time $13.7 \mathrm{~s}$, alanine aminotransferase $196 \mathrm{U} / \mathrm{L}$, aspartate aminotransferase $286 \mathrm{U} / \mathrm{L}$, lactate dehydrogenase $739 \mathrm{U} / \mathrm{L}$, creatine kinase $1092 \mathrm{U} / \mathrm{L}$, creativekinase MB $78 \mathrm{U} / \mathrm{L}$, and cardiac troponin I $0.01 \mathrm{ng} / \mathrm{mL}$. The patient did not have hypoxemia and the arterial blood gas analyses were normal. He was treated in the intensive care unit. About fifteen hours later, he became somnolence and gradually developed into a coma. Neurological examination revealed bilateral Babinski sign. His pupils were isocoric and bilaterally responsive to light. There were not cutaneous manifestations. Blood gas analysis was retested and normal. Echocardiogram showed normal ventricular function without any thrombus or patent foramen ovale (PFO). Bilateral lower limb vascular color Doppler ultrasound did not show any thrombus signs. Although in the present case there were no remarkable intracranial abnormalities on the initial brain CT findings [Figure 3], the typical brain magnetic resonance imaging (MRI) findings of starfield patterns were observed. Both T2-weighted imaging and diffusion weighted imaging of MRI indicated multiple foci lesions in the bilateral cerebrum hemisphere white matter, grey matter, basal ganglia, corpus callosum and thalamus indicative of acute infarcts [Figure 4]. However, there was not any microbleeding on the susceptibility-weighted imaging sequences of brain MRI [Figure 4]. With the above findings, the diagnosis of the case was cerebral fat embolism syndrome. The patient was treated with methylprednisolone injection (80 mg, intravenous infusion, twice a day) and low molecular weight heparin calcium injection (Dalteparin Sodium, Fragmin: $0.2 \mathrm{~mL} / 5000$ IU, subcutaneous injection, once a day), antibiotics (Cefoperazone sodium and sulbactam sodium) and dehydrating drugs (such as mannitol and human albumin solution). The bone fractures were firstly externally fixed upon admission into the hospital and then internally fixed on fifth day during in-hospital stay. Unfortunately, the patient did not recover and remained unconscious, and he was discharged after 7 days of hospitalization.

\section{DISCUSSION}

FES is a common clinical entity that can occasionally have significant neurological sequelae. Fat emolysm syndrome has been reported not only after long-bone injuries, but also after rib or tarsal bone involvement ${ }^{[3,4]}$. The risk of fat emboli is highest within the first few days after trauma. Clinical symptoms of fat embolism usually develop gradually within 24 to $72 \mathrm{~h}$ after injury ${ }^{[5]}$, but in some cases, early manifestation occurs. Patients can present with triad of varying severity of neurological, respiratory, and cutaneous manifestations, depending on the embolic burden in the respective vasculature. CFES is a variant of FES characterized by a predominance of neurologic manifestations often without the pulmonary or dermatologic findings seen in FES. The period from the time of injury to the development of cerebral FES is typically between $12 \mathrm{~h}$ and 3 days ${ }^{[6]}$. 


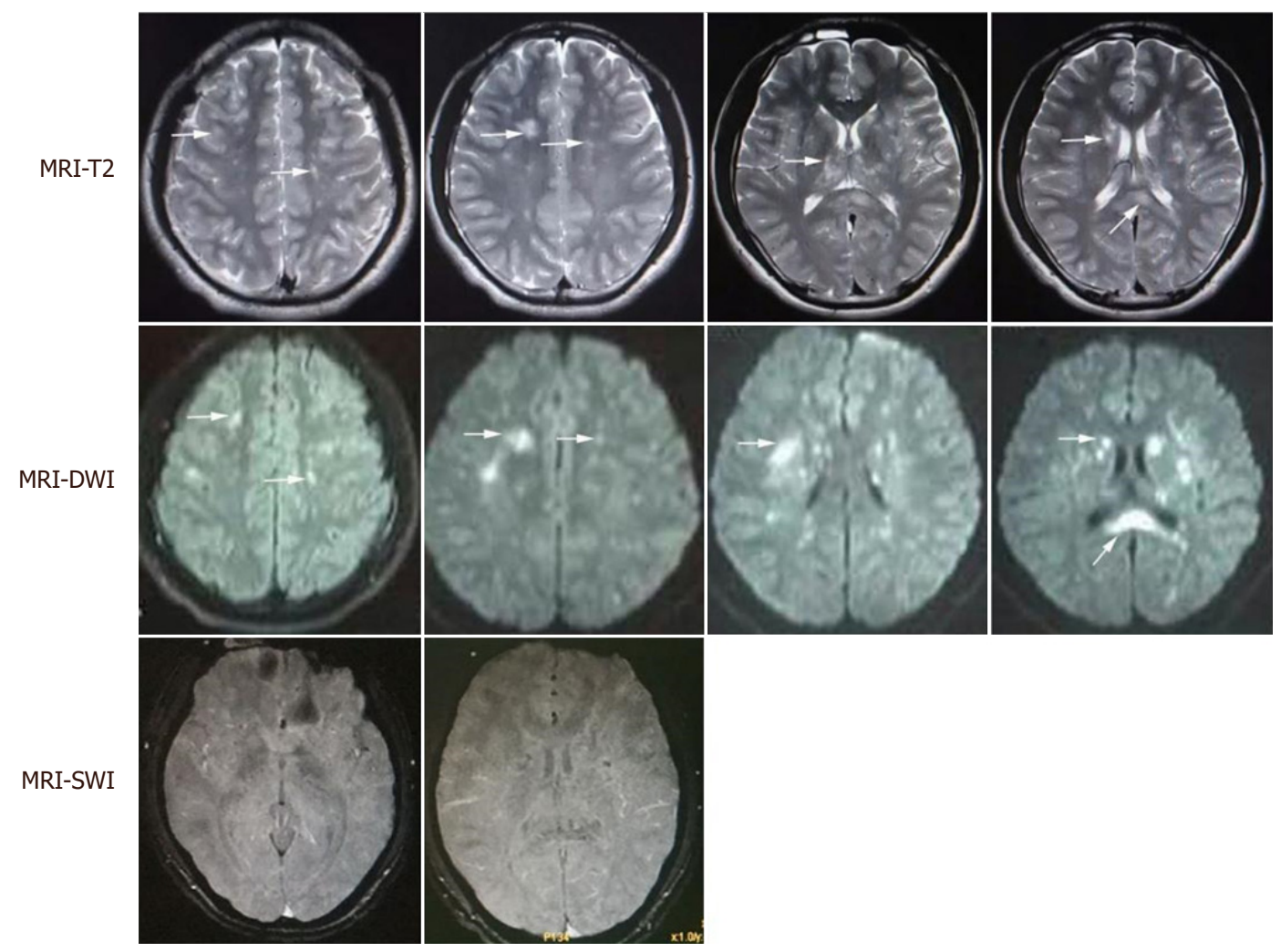

Figure 4. Both $\mathrm{T}_{2}$-weighted imaging and diffusion weighted imaging (DWI) of magnetic resonance imaging (MRI) indicated multiple foci lesions in the bilateral cerebrum hemisphere white matter, grey matter, basal ganglia, corpus callosum and thalamus indicative of acute infarcts (arrow). However, there was not any microbleeding on the susceptibility-weighted imaging sequences of brain MRI

CFES occurs after fat emboli enter the arterial circulation. However, the underlying mechanism remains poorly understood. It was suggested that fat globules may enter the arterial circulation by two mechanisms ${ }^{[2,7]}$. Firstly, fat globules can enter the left atrium directly from the right heart through a shunt, such as a PFO (paradoxical embolism), especially when the patient takes a deep breath or performs Valsalva maneuver after trauma. Secondly, microglobules of fat may filter directly through the lung capillaries to reach the arterial system. The diameter of these microemboli are small (7-10 $\mu \mathrm{m}$ in diameter) and malleable and may not lead to significant pulmonary injury ${ }^{[7]}$. In our case, the absence of PFO and arrhythmia in this patient with CFE supports the latter mechanism. However, the mechanisms underlying the pathophysiology of CFE are complex. Great amounts of microglobules of fat may randomly enter the internal carotid artery or/and vertebrobasilar artery, and usually cause distal vascular occlusion. Simple vascular occlusion with ischemia probably does not fully explain the FES. Mechanical mobilization of fat globules from the bone marrow to the intramedullary veins and pulmonary microcirculation, along with endothelial damage by fat metabolism products and cytokines, has been proposed ${ }^{[8]}$. Such biochemical pathways of injury result from the complex inflammatory response to free fatty acids released by the hydrolysis of embolized fat that has entered blood vessels. This inflammatory response may have local effects on the brain and other tissues and systemic consequences, including shock and anticoagulation ${ }^{[2,7]}$.

Neurologic manifestations of CFES vary greatly, ranging from mild headache, diffuse encephalopathy, and seizures to focal features, including pyramidal signs, pupillary paresis, and aphasia ${ }^{[1,2,7]}$. These signs can occur in isolation or accompany with respiratory and cutaneous manifestations. Some patients do not have hypoxemia, and these petechiae occur in only $20 \%-50 \%$ of patients and resolve quickly. Therefore, it is difficult for us to diagnose the case. The Gurd's and Wilson's criteria are often used to make diagnoses ${ }^{[9]}$. 
One major and four minor criteria must be present to formally diagnose FES. Neither of these diagnostic tools includes brain imaging, which seems to be the most specific. CFES is a clinical diagnosis, but specific findings on neuroimaging studies can be strongly supportive. The purpose of a CT scan is to rule out certain stroke mimics and detect hemorrhage, not necessarily to rule in the diagnosis of ischemic stroke. CT scans may not be sensitive enough to detect an ischemic stroke, especially if it is small, acute (especially within $24 \mathrm{~h}$ of the stroke onset), or in the posterior fossa (i.e., brainstem and cerebellum areas). In other words, a normal CT scan does not rule out the diagnosis of ischemic stroke. Noticeably, it is important to underline that a careful examination of brain-CT findings, such as the topography and density measurements of round lesions (such as round hypodense lesions, $-40 \mathrm{HU})^{[3]}$, were enough to confirm the clinical suspicion of $\mathrm{CFE}^{[3]}$. This is important because in some cases the MRI cannot be available or would be impossible to perform. MRI is more sensitive and demonstrates multiple small hyperintense, intracerebral lesions. There were great amounts of hyperintense lesions on MRI T2-weighted scans. The most characteristic MRI finding is the starfield pattern, demonstrating scattered foci of high-intensity restricted diffusion on diffusion-weighted imaging ${ }^{[7,10]}$. This is most apparent in the acute phase, from $4 \mathrm{~h}$ to the first few days from the time of injury. Such widespread petechial hemorrhage and bland microinfarction have been demonstrated on autopsy ${ }^{[11]}$. In our case, the initial brain-CT scan was normal, while MRI showed extensive cortical and subcortical regions fat embolism which led to disturbance of consciousness. There are few differential diagnoses of disseminated hyperintense lesions on T2-weighted scans which include diffuse axonal injury, areas of vasogenic edema associated with microinfarcts, and demyelinating diseases ${ }^{[12]}$ which were ruled out by history and clinical scenario, in our case. Of note, in some patients who sustained severe trauma, both CFE and diffuse axonal injury (DAI) could be the cause of altered consciousness in the absence of marked intracranial lesions in cranial $\mathrm{CT}^{[13]}$. However, distinguishing CFE and DAI can be difficult clinically. Generally, DAI develops immediately after the insult, whereas CFE occurs 24 to $72 \mathrm{~h}$ after the trauma and even after internal fixation for the fractures ${ }^{[13]}$. It was reported that there was no significant difference between diagnostic performance of diffusion tensor imaging (DTI) and conventional MRI in CFES, but a difference in directional diffusivities was clearly identified between CFES and DAI.

There are currently no disease-specific treatment guidelines for FES or CFES other than supportive care to address both intrinsic lung pathology and airway protection in the setting of neurological impairment ${ }^{[2]}$. Pharmacological intervention, including administration of heparin, dextran, aspirin, statin, albumin, and steroids and glucose loading, proved to be ineffective ${ }^{[14,15]}$. Corticosteroids have been extensively studied with variable results, and their use is controversial. In cases of fulminant FES, corticosteroids may be considered. Gupta et al ${ }^{[16]}$ propose a regimen of methylprednisolone $1.5 \mathrm{mg} / \mathrm{kg}$ IV every $8 \mathrm{~h}$ for 6 doses in a select group of patients with long bone or pelvic fractures at high risk of developing FES and without significant contraindications. In our case, the patient was treated with methylprednisolone injection ( $80 \mathrm{mg}$ twice a day). The side effects of corticosteroids such as promoting coagulation and ulcer, disorder of electrolyte metabolism should be emphasized. Anticoagulation has been shown to prevent stroke in patients with cardioembolic and other noncardioembolic sources. However, the early use of anticoagulants has been associated with hemorrhagic transformation. Hitherto, there is insufficient evidence to support that routine administration of anticoagulation agent is effective and safe for FES. One possible benefit from anticoagulation for fat embolism may potentially decrease the risk of deep vein thrombosis. Early surgical stabilization should be considered. Early fixation of fractures within $24 \mathrm{~h}$ has been recommended to prevent further trauma at the injury site, thus decreasing the incidence of FES. The prognosis of CFES is variable, depending on the severity of the manifestations and on the quality and timing of treatment. Most of patients recovered fully from this disease, and other survivors remained in cognitive disorder, and some even die $\mathrm{i}^{[3,7,15]}$.

In summary, we highlight that CFES could develop within hours after long bone fractures. Neurologic manifestations of CFES vary greatly. Neuroimaging is critical in the diagnosis of CFES. The brain-CT scan indicating the presence of round, hypodense lesions within the range of fat $(-40 \mathrm{HU})$ suggests fat embolism. 
The typical starfield pattern may be present on MRI in some cases of acute phase. Supportive respiratory and neurological cares remain the mainstay of therapy. Prognosis is variable depending on the context of concomitant illness and premorbid functional status.

\section{DECLARATIONS}

\section{Authors' contributions}

Conception and design of study, first draft and revision of manuscript: Chen XY, Fan JM

Provided some clinical data: Deng MF

Provided the images data: Jiang T, Luo F

Read and approved the final manuscript: all authors

\section{Availability of data and materials}

The data and material could be available to readers upon request.

\section{Financial support and sponsorship}

This study was supported by the grants from the National Natural Science Foundation of China (81771250), Fujian Province Natural Science Fund (2016J01432), Project for Scientific and Technological Innovation of Fujian Province (2017Y9065), and Young and Middle-aged Talents Training Project of Health and Family Planning Committee of Fujian Province (2015-ZQN-JC-5).

\section{Conflicts of interest}

All authors declared that there are no conflicts of interest.

\section{Ethical approval and consent to participate}

The study was approved by the local ethics committee and informed consent was obtained from the patient's guardian.

\section{Consent for publication}

Not applicable.

\section{Copyright}

(c) The Author(s) 2018.

\section{REFERENCES}

1. Kosova E, Bergmark B, Piazza G. Fat embolism syndrome. Circulation 2015;131:317-20.

2. Mijalski C, Lovett A, Mahajan R, Sundararajan S, Silverman S, Feske S. Cerebral fat embolism: a case of rapid-onset coma. Stroke 2015;46:e251-3.

3. Scarpino M, Lanzo G, Moretti M, Olivo G, Amantini A, Grippo A. Delayed cerebral fat embolism occurring after off-pump coronary artery bypass grafting. Cardiol J 2018;25:155-7.

4. Kralovec ME, Houdek MT, Martin JR, Morrey ME, Cross WR. Atypical presentation of fat embolism syndrome after gunshot wound to the foot. Am J Orthop (Belle Mead NJ) 2015;44:E71-4.

5. Jorens PG, Van Marck E, Snoeckx A, Parizel PM. Nonthrombotic pulmonary embolism. Eur Respir J 2009;34:452-74.

6. Manousakis G, Han DY, Backonja M. Cognitive outcome of cerebral fat embolism. J Stroke Cerebrovasc Dis 2012;21:901-6.

7. Kellogg RG, Fontes RB, Lopes DK. Massive cerebral involvement in fat embolism syndrome and intracranial pressure management. J Neurosurg 2013;119:1263-70.

8. Taviloglu K, Yanar H. Fat embolism syndrome. Surg Today 2007;7:5-8.

9. Aggarwal R, Pal S, Soni KD, Gamangatti S. Massive cerebral fat embolism leading to brain death: a rare presentation. Indian J Crit Care Med 2015;19:687-9.

10. Herway ST, Slotto J, Harlan E, Newhouse B. Cerebral fat embolism syndrome. Anesthesiology 2016;124:1167.

11. Miller P, Prahlow JA. Autopsy diagnosis of fat embolism syndrome. Am J Forensic Med Pathol 2011;32:291-9.

12. Parizel PM, Demey HE, Veeckmans G, Verstreken F, Cras P, Jorens PG, De Schepper AM. Early diagnosis of cerebral fat embolism 
syndrome by diffusion-weighted MRI (starfield pattern). Stroke 2001;32:2942-4.

13. Chen PC, Hsu CW, Liao WI, Chen YL, Ho CH. Hyperacute cerebral fat embolism in a patient with femoral shaft fracture. Am J Emerg Med 2013;31:1420.e1-3.

14. Whalen LD, Khot SP, Standage SW. High-dose rosuvastatin treatment for multifocal stroke in trauma-induced cerebral fat embolism syndrome: a case report. Pediatr Neurol 2014;51:410-3.

15. Mijalski C, Lovett A, Mahajan R, Sundararajan S, Silverman S, Feske S. Cerebral fat embolism. Stroke 2015;46:e251-3.

16. Gupta A, Reilly CS. Fat embolism. CEACCP 2007;7:148-51. 